\title{
Correction to: Hyperprogressive disease and its clinical impact in patients with recurrent and/or metastatic head and neck squamous cell carcinoma treated with immune-checkpoint inhibitors: Korean cancer study group HN 18-12
}

\author{
Ji Hyun Park ${ }^{1}$. Sang Hoon Chun ${ }^{2}$. Yun-Gyoo Lee ${ }^{3} \cdot$ Hyun Chang ${ }^{4} \cdot$ Keun-Wook Lee ${ }^{5}$ Hye Ryun Kim ${ }^{6}$. \\ Seong Hoon Shin ${ }^{7} \cdot$ Ho Jung An ${ }^{8} \cdot$ Kyoung Eun Lee ${ }^{9} \cdot$ In Gyu Hwang ${ }^{10} \cdot$ Myung-Ju Ahn ${ }^{11}$. Sung-Bae Kim ${ }^{12}$. \\ Bhumsuk Keam ${ }^{13}$ (1)
}

Published online: 27 August 2020

(c) Springer-Verlag GmbH Germany, part of Springer Nature 2020

\section{Correction to: \\ Journal of Cancer Research and Clinical Oncology https://doi.org/10.1007/s00432-020-03316-5}

In the original article published, the first name of the author is incorrect. The correct author name is Ji Hyun Park.

The original article can be found online at https://doi.org/10.1007/ s00432-020-03316-5.

Sung-Bae Kim

sbkim3@amc.seoul.kr

$\triangle$ Bhumsuk Keam

bhumsuk@snu.ac.kr

Hye Ryun Kim

NOBELG@yuhs.ac

1 Department of Internal Medicine, Konkuk University Medical Center, Konkuk University School of Medicine, Seoul, Republic of Korea

2 Department of Internal Medicine, Bucheon St. Mary's Hospital, College of Medicine, The Catholic University of Korea, Bucheon, Republic of Korea

3 Department of Internal Medicine, Kangbuk Samsung Hospital, Sungkyunkwan University School of Medicine, Seoul, Republic of Korea

4 Department of Internal Medicine, International St. Mary's Hospital, Catholic Kwandong University College of Medicine, Incheon, Republic of Korea

5 Department of Internal Medicine, Seoul National University Bundang Hospital, Seongnam, Republic of Korea

6 Department of Internal Medicine, Yonsei Cancer Center, Yonsei University College of Medicine, Seoul, Republic of Korea
Publisher's Note Springer Nature remains neutral with regard to jurisdictional claims in published maps and institutional affiliations.
7 Department of Internal Medicine, Kosin University Gospel Hospital, Kosin University College of Medicine, Busan, Republic of Korea

8 Department of Internal Medicine, St. Vincent's Hospital, College of Medicine, The Catholic University of Korea, Suwon, Republic of Korea

9 Department of Hematology and Oncology, Ewha Woman's University Hospital, Seoul, Republic of Korea

10 Department of Internal Medicine, Chung-Ang University Hospital, Chung-Ang University College of Medicine, Seoul, Republic of Korea

11 Department of Internal Medicine, Samsung Medical Center, Sungkyunkwan University School of Medicine, Seoul, Republic of Korea

12 Department of Internal Medicine, Department of Oncology, Asan Medical Center, University of Ulsan College of Medicine, 88 Olympic-ro 43-gil, Songpa-gu, Seoul 05505, Republic of Korea

13 Department of Internal Medicine, Seoul National University Hospital, 101 Daehak-ro, Jongno-gu, Seoul 03080, Republic of Korea 